\title{
Chronic renal dysfunction in maintenance heart transplant patients: the ICEBERG study
}

\author{
F. González-Vílchez ${ }^{\mathrm{a}}$ J.M. Arizón ${ }^{\mathrm{b}}$,J. Segovia ${ }^{\mathrm{c}}$, L. Almenar ${ }^{\mathrm{d}}$, M.G. Crespo- \\ Leiro $^{\mathrm{e}}$, J. Palomo ${ }^{\mathrm{f}}$, J.F. Delgado ${ }^{\mathrm{g}}$, S. Mirabet ${ }^{\mathrm{h}}$, G. Rábago ${ }^{\mathrm{i}}$, F. Pérez-Villa ${ }^{\mathrm{j}}$, B. \\ Díaz $^{\mathrm{k}}$, M.L. Sanz ${ }^{1}$ D. Pascual ${ }^{\mathrm{m}}$, L. de la Fuente ${ }^{\mathrm{n}}$, G. Guinea ${ }^{\mathrm{o}}$ on behalf of the \\ ICEBERG Study Group
}

\begin{abstract}
${ }^{a}$ Hospital Marqués de Valdecilla, Santander, Spain; ${ }^{b}$ Hospital Reina Sofía, Córdoba, Spain; ${ }^{c}$ Hospital Puerta de Hierro, Madrid, Spain; ${ }^{d}$ Hospital La Fe, Valencia, Spain; e Hospital Juan Canalejo, A Coruña, Spain; ${ }^{f}$ Hospital Gregorio Marañón, Madrid, Spain; ${ }^{g}$ Hospital Doce de Octubre, Madrid, Spain; ${ }^{h}$ Hospital de la Santa Creu $i$ Sant Pau, Barcelona, Spain; ${ }^{i}$ Clínica Universitaria de Navarra, Pamplona, Spain; ${ }^{j}$ Hospital Clínic i Provincial de Barcelona, Barcelona, Spain; ${ }^{k}$ Hospital Central de Asturias, Oviedo, Spain; Hospital Universitario Miguel Servet, Zaragoza, Spain; ${ }^{m}$ Hospital Virgen de la Arrixaca, Murcia, Spain; ${ }^{n}$ Hospital Clínico Universitario de Valladolid, Valladolid, Spain; ${ }^{\circ}$ Medical Department Novartis, Madrid, Spain
\end{abstract}

\begin{abstract}
Chronic renal dysfunction (CRD) is a major complication after heart transplantation. We sought to describe the renal function over time, to assess the risk factors associated with CRD development, and to evaluate the clinical attitudes on diagnosis and treatment of CRD. A retrospective, cross-sectional, multicenter study was conducted in 13 outpatient clinics in Spain. A total of 244 heart recipients who survived more than 2 years after transplantation were included. Post-transplantation follow-up was 7.7 years (range: 2-22 years). CRD was diagnosed in $32.4 \%$ of patients at a mean of 3.3 years after transplantation. Serum creatinine increased $0.1 \pm 0.2 \mathrm{mg} / \mathrm{dL}$ per year in CRD group compared with $0.0 \pm 0.2 \mathrm{mg} / \mathrm{dL}$ per year in non-CRD group $(P=$ .003 ) and glomerular filtration rate decreased $-1.5 \pm 4.3 \mathrm{~mL} / \mathrm{min} / 1.73 \mathrm{~m}^{2}$ per year in CRD group versus -0.1 $\pm 4.8 \mathrm{~mL} / \mathrm{min} / 1.73 \mathrm{~m}^{2}$ per year in non-CRD group $(P=.027)$. After CRD diagnosis, major changes in immunosuppression based on calcineurin inhibitors reduction were instituted in $46.8 \%$ of patients. Multivariate model identified recipient age $(P<.0001)$, female sex $(P=.0398)$, and time since transplant $(P<.0001)$ as predictors of CRD. In conclusion, the prevalence of CRD in long-term heart recipient survivors was quite high. CRD was associated with nonmodifiable factors (age, gender, and time since transplant).
\end{abstract}


At present, chronic renal dysfunction (CRD) constitutes a major cause of morbidity and mortality after heart transplantation. Overall, the incidence of CRD after transplantation of a nonrenal organ is associated with a greater than fourfold increase in mortality [1]. Thus, early detection of CRD is decisive to delay the progression of chronic kidney disease and improve the long-term outcomes.

Although post-transplant CRD has a multifactorial etiology [1], [2], [3], [4], [5], [6], [7], [8], [9], [10], [11], [12], [13], it is considered that calcineurin inhibitors (CNI)-related nephrotoxicity plays a key role [14], [15]. Estimates of the prevalence of CRD following solid organ transplants vary as a result of differences in the definitions used. For instance, the prevalence of Kidney Disease Outcome Quality Initiative (K/DOQI) guidelines [16] stage 3 or worse CRD (glomerular filtration rate [GFR] less than $60 \mathrm{~mL} / \mathrm{min} / 1.73 \mathrm{~m}^{2}$ ) among heart transplant patients was $61 \% 7$ years after transplantation [12]. When defined as a GFR less than $30 \mathrm{~mL} / \mathrm{min} / 1.73 \mathrm{~m}^{2}$ (stage 4 or worse), the 5-year risk of CRD was $11 \%$ for heart transplants [1].

The present study analyzed retrospectively the renal function in cardiac transplant recipients in 13 centers of Spain and aimed to describe the changes over time of serum creatinine and GFR levels and the clinical factors associated with the development of CRD. Additionally, we attempted to define the clinical attitudes with respect to diagnosis and therapy of CRD in real life over the study period.

\section{Patients and Methods}

The ICEBERG study was a retrospective, cross-sectional, multicenter study conducted in 13 cardiac transplantation outpatient clinics in Spain. All patients were aged 18 years or older and had a functioning cardiac allograft for at least 2 years before inclusion. Recipients of a multiorgan transplant and patients on renal replacement therapy were excluded. The study was conducted according to 2000 Declaration of Helsinki, with approval of the protocol by an Institutional Ethics Committee. All patients signed the informed consent prior to their participation.

A systematic, nonrandom sequential sampling was performed in each center during a 1-week period by the treating cardiologists participating in the study, yielding 244 patients fulfilling inclusion and exclusion criteria. Information was gathered between May and June 2007 by means of patient interviews and review of medical records. Relevant information included patient demographics and medical history, cardiac failure etiology, transplantation date, donor age and gender, recipient viral serologies, and immunosuppressive regimen used at hospital discharge after transplantation. Detailed post-transplant information was also obtained, including information on post-transplant medical history (graft rejection, graft vascular disease, diabetes mellitus, hypertension, and malignancy), diagnosis of CRD, proteinuria determinations, and information on antiproteinuric treatment (ie, angiotensin converting enzyme [ACE]) inhibitors/angiotensin receptor [AR] blockers), serum levels of creatinine, and changes in the immunosuppressive treatment. Post-transplant data were gathered at the study visit and at 5 previous different points: nadir visit (corresponding to the visit with the lowest creatinine value within the first year posttransplant), 1 year after transplantation, and three next visits (from third to fifth visit), which were distributed equally in the period between the first year visit and the study visit. Creatinine and GFR slopes from nadir $\left(\mathrm{mg} / \mathrm{dL}\right.$ per year and $\mathrm{mL} / \mathrm{min} / 1.73 \mathrm{~m}^{2}$ per year, respectively) were calculated for each patient by the linear least squares method. Finally, the investigators were requested to establish the presence of CRD according to their own clinical assessment with knowledge of serum creatinine and GFR levels, and the results were tested for the presence of a meaningful degree of renal failure, defined as GFR $\leq 45 \mathrm{~mL} / \mathrm{min} / 1.73 \mathrm{~m}^{2}$ (grade $3 \mathrm{~B}$ of the K/DOQI guidelines [16]). GFR was estimated using the abbreviated or four-variable Modification of Diet in Renal Disease equation [17], [18]. 
Categorical and continuous variables were summarized as percentages and mean \pm standard deviation, respectively. Chi-square tests were used to compare frequencies between subgroups for qualitative variables. Continuous variables were compared by Student $t$ test. The relationship between quantitative variables was assessed by Pearson correlation. To determine the factors associated with the change in GFR over time, a linear mixed-effect model with repeated measures was fitted [19]. The variables included in the model were gender, age, CNI therapy, post-transplant diabetes mellitus, post-transplant hypertension, renoprotective treatment, and time since transplantation (all of them recognized as risk factors in previous reports). All factors were included as fixed effects. A random patient intercept and identity scale covariance matrix were used. The rate of change in GFR over time was analyzed by the factor by time interaction of those variables included in the model. Significance was established at a $P$ value $\leq .05$. Data were analyzed using SPSS 12.0 statistical package (SPSS Inc, Chicago, Ill, United States).

\section{Results}

\section{Patient Characteristics}

Table 1 shows the demographic and clinical characteristics of the study patients. The vast majority of patients were men and Caucasian, with a mean age of $58.9 \pm 12.0$ years at the time of study visit. Ischemic and idiopathic cardiomyopathies were the most frequent causes leading to cardiac transplantation. Seventy-three per cent of the recipients were positive for cytomegalovirus serology. Antibody induction therapy was used in $70 \%$ of patients. The most frequently used immunosuppressants were cyclosporine $(81.6 \%)$ and azathioprine $(57.8 \%)$. Acute rejection of International Society for Heart and Lung Transplantation grade $\geq$ IIIA had been diagnosed in $46 \%$ of patients, and the mean number of rejection episodes was $2.5 \pm 2.5$. Twenty percent of patients developed a malignancy, and $15.8 \%$ were diagnosed with graft vascular disease. Serious infections occurred in one third of the patients. Diabetes mellitus was present in $25 \%$ of patients. Hypertension was detected in $47.5 \%$ of patients. The mean time between transplantation and study visit was $7.7 \pm 4.0$ years (range: $2-22$ years). Visit 3 was carried out at a mean of $2.9 \pm 1.8$ years post-transplant, visit 4 at a mean of $4.6 \pm 2.5$ years post-transplant, and visit 5 at $6.3 \pm 3.3$ years post-transplant. 
Table 1. Demographics and Clinical Characteristics of Heart Recipients With or Without Diagnosis of Chronic Renal Dysfunction (79 and 165 Patients, Respectively)

Patients with CRD, $n(\%) \quad$ Patients without CRD, $n(\%) \quad$ Total, $n(\%)$

\begin{tabular}{|c|c|c|c|}
\hline $\operatorname{Age}(y)$, mean $\pm \mathrm{SD} \underline{*}$ & $63.2 \pm 10.4$ & $56.8 \pm 12.1$ & $58.9 \pm 12.0$ \\
\hline Gender (male) & $68(86.1)$ & $129(78.2)$ & $197(80.7)$ \\
\hline Race (Caucasian) & $78(98.7)$ & $163(98.8)$ & $241(98.8)$ \\
\hline \multicolumn{4}{|l|}{ Reason for transplantation } \\
\hline Ischemic cardiomyopathy & $43(54.4)$ & $55(33.3)$ & $98(40.2)$ \\
\hline Dilated cardiomyopathy & $21(26.6)$ & $73(44.2)$ & $94(38.5)$ \\
\hline Other & $64(81.0)$ & $128(77.6)$ & $52(21.3)$ \\
\hline Cytomegalovirus serology (positive) & $59(74.7)$ & $119(72.1)$ & $178(72.9)$ \\
\hline Induction therapy $\underline{*}$ & $58(73.4)$ & $113(68.5)$ & $171(70.0)$ \\
\hline Anti-CD25 & $21(25.6)$ & $36(21.8)$ & $57(23.4)$ \\
\hline OKT3 & $18(22.8)$ & $66(40.0)$ & $84(34.4)$ \\
\hline Thymoglobulin & $19(24.0)$ & $11(6.7)$ & $30(12.3)$ \\
\hline \multicolumn{4}{|l|}{ Immunosuppressant therapy (at discharge) } \\
\hline Cyclosporine & $65(82.3)$ & $134(81.2)$ & 199 (81.6) \\
\hline Tacrolimus & $15(19.0)$ & $28(17.0)$ & $43(17.7)$ \\
\hline Mycophenolate mofetil & $31(39.2)$ & $66(40.0)$ & $97(39.8)$ \\
\hline Azathioprine & $44(55.7)$ & $97(58.8)$ & $141(57.8)$ \\
\hline Mycophenolic sodium & $0(0.0)$ & $1(0.6)$ & $1(0.4)$ \\
\hline Everolimus & $0(0.0)$ & $2(2.5)$ & $2(0.8)$ \\
\hline Sirolimus & $1(1.3)$ & $4(2.4)$ & $5(2.1)$ \\
\hline Prednisone & $76(96.2)$ & $155(93.9)$ & $231(94.7)$ \\
\hline \multicolumn{4}{|l|}{ Concomitant therapies } \\
\hline Alpha-blockers & $13(6.4)$ & $15(4.0)$ & $28(11.4)$ \\
\hline Beta-blockers & $13(6.4)$ & $11(3.0)$ & $24(9.8)$ \\
\hline Calcium channel blockers & $30(14.8)$ & $66(17.7)$ & $96(39.3)$ \\
\hline ACE inhibitors/AR blockers & $42(53.2)$ & $79(47.9)$ & $121(49.5)$ \\
\hline Diuretics & $36(28.4)$ & $47(28.4)$ & $83(34.0)$ \\
\hline Statins & $63(31.0)$ & $137(36.8)$ & $200(81.9)$ \\
\hline None & $6(3.0)$ & $17(4.6)$ & $23(9.4)$ \\
\hline Acute rejection (ISHLT grade $\geq$ IIIA) & $38(48.1)$ & $74(44.8)$ & $112(45.9)$ \\
\hline Graft vascular disease & $10(12.7)$ & $26(15.8)$ & $36(14.8)$ \\
\hline Malignancy & $21(26.6)$ & $26(15.8)$ & $47(19.2)$ \\
\hline Diabetes mellitus post-transplant $\underline{\text { }}$ & $23(29.1)$ & $38(23.0)$ & $61(25.0)$ \\
\hline Hypertension post-transplant & $44(55.7)$ & $72(43.6)$ & $116(47.5)$ \\
\hline Serious infection & $29(36.7)$ & $52(31.5)$ & $81(31.1)$ \\
\hline Opportunistic infection & $17(21.5)$ & $27(16.4)$ & $44(18.0)$ \\
\hline Time since transplantation $(\mathrm{y})$, mean $\pm \mathrm{SD}$ & $7.7 \pm 4.1$ & $7.7 \pm 3.9$ & $7.7 \pm 4.0$ \\
\hline
\end{tabular}

ACE, angiotensin converting enzyme; AR, angiotensin receptor; ISHLT, International Society for Heart and Lung Transplantation; CRD, chronic renal dysfunction; SD, standard deviation.

* Differences between groups were statistically significant according to: age $(P<.0001)$, induction therapy $(P=.0003)$, and diabetes mellitus post-transplant $(P=.0203)$. 
For the whole group, the evolution of renal function is summarized in Fig 1. GFR mirrored serum creatinine levels and both showed deterioration in renal function within the first year after transplantation from near normal values. At this point, approximately one fifth of patients had at least grade 3B renal failure. After the first year, renal function only showed a mild decrease, although by 8 years after transplantation, nearly one third of patients had moderate to severe renal failure (GFR $\left.<45 \mathrm{~mL} / \mathrm{min} / 1.73 \mathrm{~m}^{2}\right)$.

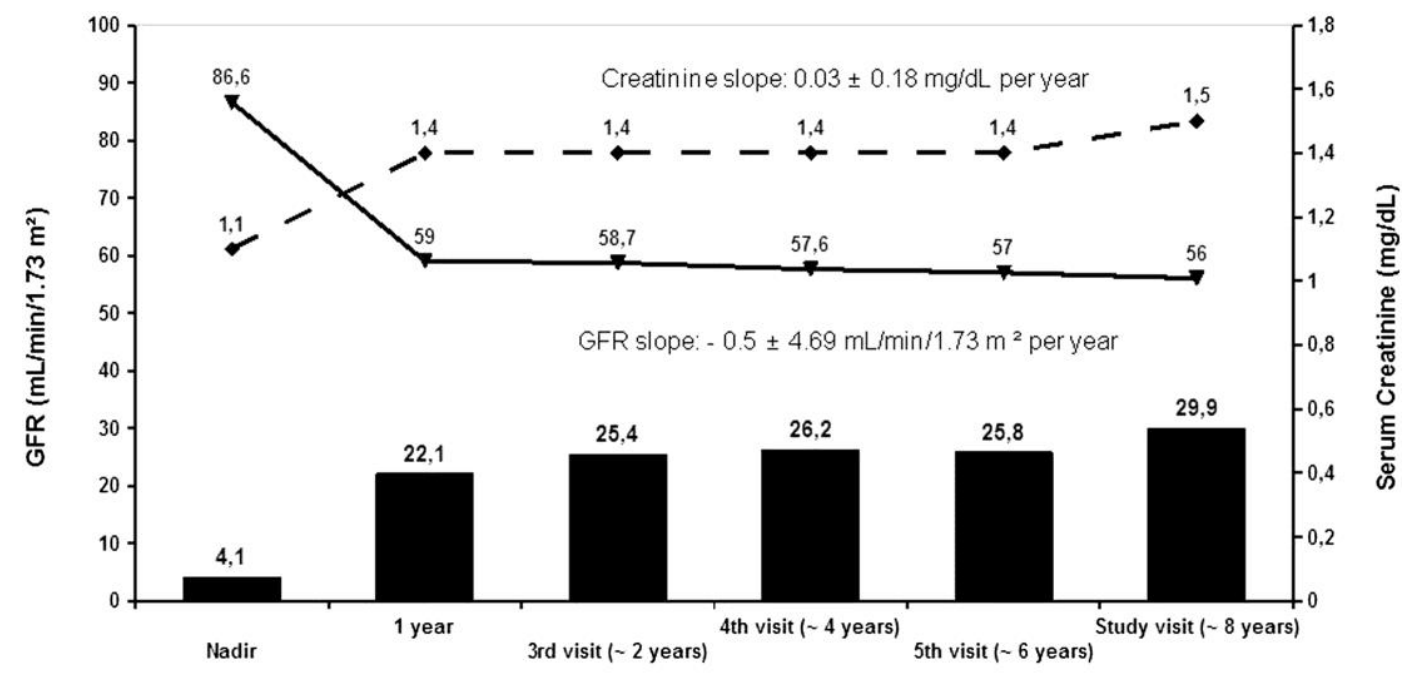

Time after transplantation

Fig 1. Evolution of renal function according to serum creatinine levels and glomerular filtration rate (GFR) in 242 chronic heart transplant recipients. Solid columns represents the percentage of patients with GFR $<45 \mathrm{~mL} / \mathrm{min} / 1.73 \mathrm{~m}^{2}$.

The factors associated with the change rate in GFR over time are summarized in Table 2. The most relevant effect related to a best renal function was the male gender as compared to female gender $(P=.0398)$. The factors related with a worse evolution in renal function were age at transplantation $\left(-0.7 \mathrm{~mL} / \mathrm{min} / 1.73 \mathrm{~m}^{2}\right.$ for each additional year, $\left.P<.0001\right)$, and time since transplantation $(P<.0001)$. On the contrary, there were no differences related to the presence of diabetes mellitus post-transplant $(P=.1653)$, hypertension post-transplant $(P=.1779)$, CNI therapy $(P=.1021)$, or renoprotective treatment $(P=.1740)$. 
Table 2. Linear Mixed-Effects Model for Response of Renal Function

\begin{tabular}{lcc}
\hline Factor & $\mathrm{F}$ & $P$ value \\
\hline & & \\
Intercept & 120.13 & $<.0001$ \\
Recipient gender (male vs female) & 5.85 & .0398 \\
Age & -0.67 & $<.0001$ \\
CNI therapy (yes vs no) & 1.64 & .1021 \\
Diabetes mellitus post-transplant (yes vs no) & -3.57 & .1653 \\
Hypertension post-transplant (yes vs no) & -3.03 & .1779 \\
Renoprotective treatment (no vs yes) & -1.59 & .1740 \\
Time since transplantation & & \\
1-y visit vs nadir & -27.29 & $<.0001$ \\
3rd visit $(\sim 2$ y) vs nadir & -28.56 & $<.0001$ \\
4th visit $(\sim 4$ y) vs nadir & -29.59 & $<.0001$ \\
5th visit $(\sim 6$ y) vs nadir & -29.57 & $<.0001$ \\
Study visit $(\sim 8$ y) vs nadir & -30.28 & $<.0001$ \\
& & \\
\hline
\end{tabular}

CNI, calcineurin inhibitor.

CRD was clinically diagnosed by treating physicians in 79 out of 244 patients $(32.4 \%)$. The mean time until physicians considered the diagnosis of CRD was $39.5 \pm 42.8$ months after transplantation. Patients with the diagnosis of CRD showed steeper creatinine $(0.1 \pm 0.2 \mathrm{mg} / \mathrm{dL}$ per year) and GFR slopes $\left(-1.5 \pm 4.3 \mathrm{~mL} / \mathrm{min} / 1.73 \mathrm{~m}^{2}\right.$ per year) as compared to those patients without CRD $\left(0.0 \pm 0.2 \mathrm{mg} / \mathrm{dL}\right.$ per year, $P=.0003$; and $-0.1 \pm 4.8 \mathrm{~mL} / \mathrm{min} / 1.73 \mathrm{~m}^{2}$ per year, $P=.027$ respectively; Fig 2). A steady increase in serum creatinine levels was observed in patients with CRD from the time of transplantation, whereas they remained relatively stable over time in those patients without CRD. In turn, changes in GFR showed similar evolution in both groups. We observed a steep decline within 1 year after transplantation and a stable course thereafter, although patients with CRD tended to have a higher decline in GFR as compared to those patients without CRD over this later period. Nadir creatinine serum levels were not different between CRD and non-CRD patients. Creatinine curves commenced to separate between groups beyond the first year after transplantation (Fig 2A). In contrast to creatinine levels, differences for GFR between CRD and non-CRD patients could be observed from the nadir point (Fig 2B). Figure 3 summarizes the proportion of patients having a GFR $\leq 45 \mathrm{~mL} / \mathrm{min} / 1.73 \mathrm{~m}^{2}$ according to the clinical diagnosis of CRD. At the study visit, more than two thirds of patients had at least grade $3 \mathrm{~B}$ renal failure. Noteworthy, $10 \%$ of patients without CRD according to physician's criteria had moderate to severe decline in GFR. Differences between CRD and non-CRD patients were significant for age at the study visit $(P<.0001)$, antibody induction at the time of transplantation $(P=.0003)$, and posttransplant diabetes $(P=.02$; Table 1$)$. 

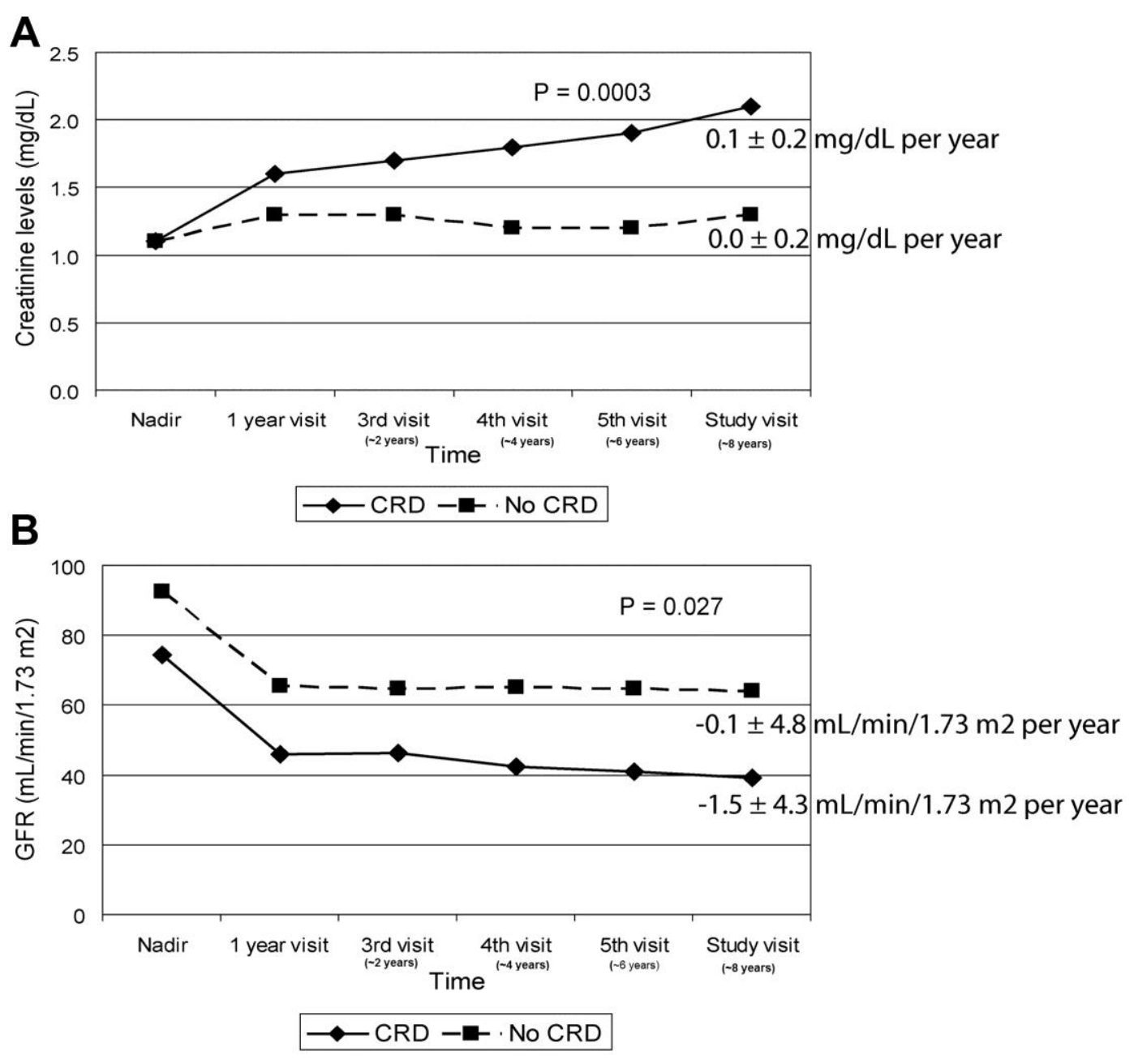

Fig 2. Evolution of serum creatinine levels (A) and glomerular filtration rate (GFR) (B) in 242 chronic heart recipients with chronic renal dysfunction (CRD; solid line, rhombus marks) and with no renal dysfunction (dashed line; square marks). Creatinine and GFR slopes from nadir are shown on the right. Time since transplant (years) for third visit, fourth visit, fifth visit, and study visit corresponds roughly to $2,4,6$, and 8 years, respectively. 


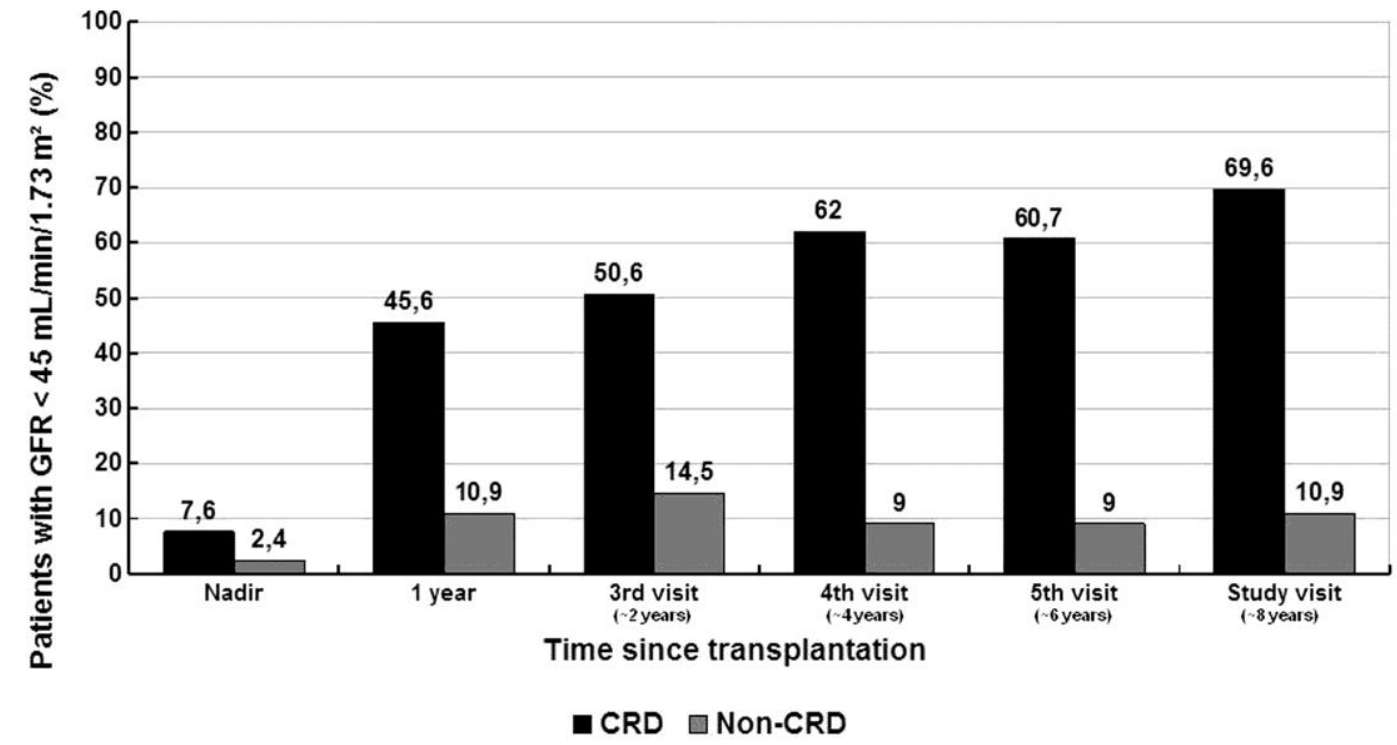

Fig 3. Percentage of patients with glomerular filtration rate $<45 \mathrm{~mL} / \mathrm{min} / 1.73 \mathrm{~m}^{2}$ in patients with and without clinical diagnosis of chronic renal dysfunction (CRD).

\section{Therapy Changes After Clinical Diagnosis of CRD}

In $83.5 \%$ of the cases, the treating physicians considered calcineurin nephrotoxicity as the main cause of CRD. Changes in immunosuppressive therapy were carried in 37 of 79 patients (46.8\%). All changes were based on the reduction of CNI. Major changes included also modifications of mycophenolic acid (MPA) therapy or introduction of proliferation signal inhibitors, which were carried out in 16 patients. Patients with major changes showed significantly higher creatinine slope compared with those with isolated CNI reduction $(P=.028$; Table 3$)$.

Table 3. Changes in Immunosuppressive Therapy After the Diagnosis of Chronic Renal Dysfunction in 79 Heart Recipients

\begin{tabular}{lcc}
\hline Change & $n(\%)$ & $\begin{array}{c}\text { Creatinine slope (mg/dL per year), mean } \\
(95 \% \text { CI })\end{array}$ \\
\hline & & $0.03(-0.05-0.11)$ \\
CNI reduction and no other changes & $21(26.5)$ & $0.21(0.09-0.34)$ \\
CNI reduction/withdrawal + MPA (MMF/MPS) modification & $8(10.2)$ & $0.19(0.07-0.32)$ \\
CNI reduction/withdrawal + PSI introduction & $8(10.2)$ & $0.06(0.0-0.12)$ \\
No changes & $42(53.1)$ & \\
\hline
\end{tabular}

CI, confidence interval; CNI, calcineurin inhibitor; MPA, mycophenolic acid; MMF, mycophenolate mofetil; MPS, mycophenolate sodium; PSI, proliferation signal inhibitor. 
Assessment of proteinuria was carried out only in 4 patients. After CRD diagnosis, ACE inhibitor/AR blocker therapy was introduced in 13 patients $(16.5 \%)$. However, there were no differences between CRD group (53.1\%) and non-CRD group $(47.8 \% ; P=.49)$ for the use of these drugs at the end of follow-up.

\section{Discussion}

Although significant improvements have been made in the survival rate in heart transplantation, cardiac recipients are still subjected to serious complications, particularly renal failure. As renal function deteriorates since time from transplantation [10], [12], we first sought to assess the temporal course of renal function worsening over time. Overall, we observed a steep decline in renal function within the first year after transplantation, followed by a slow rate of deterioration over the next years. The initial worsening of renal function could be related with the high CNI serum levels maintained in this period [20] but also to the high prevalence of potential nephrotoxic conditions, such as infections and allograft rejections. As previously reported [3], [21], renal dysfunction at 1 year after transplantation is a powerful and independent predictor of $\mathrm{CRD}$ and long-term all-cause mortality in heart transplantation. The prevalence of K/DOQI 3B renal dysfunction or worse $\left(\mathrm{GFR}<45 \mathrm{~mL} / \mathrm{min} / 1.73 \mathrm{~m}^{2}\right.$ ) reached $22 \%$ at 1 year and $30 \%$ by 8 years after transplantation. These results are in between those recently reported by Crespo-Leiro et al [22] in a study of 1065 patients. They found that at 6.2 to 9.5 years after transplantation, $24 \%$ of patients had moderate renal dysfunction as defined by serum creatinine levels between 1.6 and $2.5 \mathrm{mg} / \mathrm{dL}$, whereas using GFR-based criteria $\left(30-60 \mathrm{~mL} / \mathrm{min} / 1.73 \mathrm{~m}^{2}\right)$, the prevalence of renal dysfunction was $55 \%$. Interestingly, the estimation of change over time in renal function determined by creatinine and GFR slopes displayed a similar evolution over time, which means that worsening of renal function was not due only to the aging population but to a real deterioration of renal function.

We found that the rate of change over time in GFR was independently associated with nonmodifiable factors such as age at transplant, female sex, and time since transplantation. Increasing age as a risk factor for developing renal dysfunction has been described in numerous previous studies [1], [3], [5], [6], [7], [9], [10], [12], [13]. Female sex and time since transplant were well-known risk factors for development of CRD in patients with heart transplantation, as well [1], [7], [9], [10], [12]. Comorbidities such as hypertension and diabetes were not related to the rate of change in renal function, in disagreement with previous studies [1], [4], [6], [9], [19], although there was a tendency to be associated with worsening of renal function. Remarkably, neither withdrawal of CNI therapy nor the use of renoprotective therapy (mainly ACE inhibitors) were associated with a better evolution in renal function. In fact, the creatinine slope was higher in those patients with CNI reduction or avoidance. These findings suggest that these therapy measures were carried out in patients with the most unfavorable evolution of renal function after transplantation and later, when the renal damage was irreversible [19].

This study also intended to assess the clinical attitudes with respect to the diagnosis and therapy of CRD. We found that the clinical diagnosis of renal failure was considered in quite advanced stages of renal dysfunction. In fact, in every point of the follow-up, most of the patients diagnosed with CRD by the treating physicians had GFR $<45 \mathrm{~mL} / \mathrm{min} / 1.73 \mathrm{~m}^{2}$. This has important practical implications, as the efficacy of renal-sparing strategies is probably limited to early stages of renal damage [23]. In turn, $10 \%$ of patients with no CRD at the physician's criteria had moderate to severe renal failure as assessed by GFR. This probably reflects that the clinical diagnosis of CRD in the period in which the study was performed was based on serum creatinine levels, which tends to underestimate the degree of renal failure [22]. It is also remarkable that in the present series, proteinuria, an early marker of renal damage, was only evaluated in $1.6 \%$ of patients. 
More than $80 \%$ of cases of CRD were considered to be mainly attributable to CNI-related nephrotoxicity by the investigators. Accordingly, in nearly $50 \%$ of cases, a strategy based on minimization or withdrawal of CNI therapy was attempted. This relatively low rate of modification of CNI therapy may be due to several reasons: absence of well-proven alternatives within the period in which many patients were diagnosed of CRD, and concerns about the benefitrisk ratio of therapy changes in this particular clinical context. We found the highest creatinine slopes for the patients in whom changes in CNI therapy were accompanied by introduction of MPA therapy [24], [25] or substitution for proliferation signal inhibitors [26]. This suggests that major changes in immunosuppression were used mainly in the cases with more advanced renal damage, a clinical situation where the usefulness of those changes could likely be more limited [27].

The ACE inhibitors are useful in limiting chronic damage of kidney allografts [28], a situation in which CNI therapy plays an important role. In our series, the introduction rate of ACE inhibitor/AR blocker therapy after the diagnosis of CRD was relatively low (16.5\% of patients). This finding could be attributable to three reasons: (1) physicians' concerns about a likely deterioration of an already damaged kidney; (2) a relatively high rate of previous use of ACE inhibitor/AR blocker therapy (nearly $50 \%$ of patients); and (3) the lack of proteinuria determinations.

The present study has several limitations. The inherent limitations of the retrospective, crosssectional design studies regarding the possibility of data inaccuracy, lack of information (such as pretransplant renal function or CNI target levels), and patient selection bias. Another constraint worth mentioning is that the information for this study was gathered in 2007, which means that the number of patients treated with the immunosuppressant azathioprine is much higher than in the currently used strategy, which has been now largely optimized with the introduction of MPA. On the other hand, since deceased patients and patients requiring renal replacement therapy were excluded, our study sample represents the best of the clinical scenarios for chronic heart transplantation. Moreover, the sequential recruitment and the large number of participating centers suggest that our sample is representative of heart transplant population in Spain.

In conclusion, CRD was a relatively prevalent and progressive condition in long-term heart recipient survivors. CRD was associated with nonmodifiable factors such as recipient age, gender, or time after transplantation. Thus, further efforts should be made to detect CRD early, in order to implement preventive measures at earlier stages.

\section{Acknowledgments}

We would like to thank Susana Palou, Neus Valveny, and Eva Mateu from TFS for their writing assistance support.

Investigators of the ICEBERG Study Group who participated in the study: Luis Martínez (Hospital La Fe, Valencia, Spain); María J. Paniagua (Hospital Juan Canalejo. A Coruña, Spain); Rafael Martín, José A. Vázquez de Prada (Hospital Marqués de Valdecilla, Santander, Spain); Juan Fernández-Yañez (Hospital Gregorio Marañón, Madrid, Spain); Vicenç Brossa (Hospital de la Santa Creu i Sant Pau, Barcelona, Spain); Stefano Mastrobuoni (Clínica Universitaria de Navarra, Pamplona, Spain); Eulàlia Roig (Hospital Clínic i Provincial de Barcelona, Barcelona, Spain); María Martín (Hospital Central de Asturias, Oviedo, Spain); Teresa Blasco (Hospital Universitario Miguel Servet, Zaragoza, Spain). 


\section{REFERENCES}

[1] Ojo AO, Held PJ, Port FK, et al. Chronic renal failure after transplantation of a nonrenal organ. N Engl J Med 2003;349:931.

[2] Esposito C, Semeraro L, Bellotti N, et al. Risk factors for chronic renal dysfunction in cardiac allograft recipients. Nephron 2000;84:21.

[3] Lindelow B, Bergh CH, Herlitz H, Waagstein F. Predictors and evolution of renal function during 9 years following heart transplantation. J Am Soc Nephrol 2000;11:951.

[4] Rubel JR, Milford EL, McKay DB, Jarcho JA. Renal insufficiency and end-stage renal disease in the heart transplant population. J Heart Lung Transplant 2004;23:289.

[5] Garrido IP, Crespo-Leiro MG, Paniagua MJ, et al. Independent predictors of renal dysfunction after heart transplantation in patients with normal pretransplant renal function. J Heart Lung Transplant 2005;24:1226.

[6] Al Aly Z, Abbas S, Moore E, Diallo O, Hauptman PJ, Bastani B. The natural history of renal function following orthotopic heart transplant. Clin Transplant 2005; 19:683.

[7] Diez Ojea B, Gago Gonzalez E, Diaz Corte C, et al. Study of the renal function in nonrenal organ transplantation. Transplant Proc 2006;38:2985.

[8] Sanchez Lazaro IJ, Almenar Bonet L, Martinez-Dolz L, et al. Effect of hypertension, diabetes, and smoking on development of renal dysfunction after heart transplantation. Transplant Proc 2008;40:3049.

[9] Hamour IM, Omar F, Lyster HS, Palmer A, Banner NR. Chronic kidney disease after heart transplantation. Nephrol Dial Transplant 2009;24:1655.

[10] Przybylowski P, Malyszko J, Malyszko JS. Predictors of kidney dysfunction in heart transplant recipients. Transplant Proc 2009;41:3235.

[11] Navarro-Manchon J, Martinez-Dolz L, Almenar Bonet L, et al. Predictors of renal dysfunction at 1 year in heart transplant patients. Transplantation 2010;89:977.

[12] Delgado JF, Crespo-Leiro MG, Gomez-Sanchez MA, et al. Risk factors associated with moderate-to-severe renal dysfunction among heart transplant patients: results from the CAPRI study. Clin Transplant 2010;24:E194.

[13] Zhang R, Georgiou M, Gwinner W, Zardo P, Haverich A, Bara C. Early posttransplantation hemoglobin level corresponds with chronic renal dysfunction in heart transplant recipients. Transplant Proc 2011;43:1939.

[14] Cantarovich M. Renal protective strategies in heart transplant patients. Curr Opin Cardiol 2007;22:133.

[15] Flechner SM, Kobashigawa J, Klintmalm G. Calcineurin inhibitor-sparing regimens in solid organ transplantation: focus on improving renal function and nephrotoxicity. Clin Transplant 2008;22:1.

[16] K/DOQI clinical practice guidelines for chronic kidney disease: evaluation, classification, and stratification. Am J Kidney Dis 2002;39:S1.

[17] Levey AS, Bosch JP, Lewis JB, Greene T, Rogers N, Roth D. A more accurate method to estimate glomerular filtration rate from serum creatinine: a new prediction equation. Modification of Diet in Renal Disease Study Group. Ann Intern Med 1999;130:461.

[18] Levey A, Greene T, Kusek J, Beck G, Group M. A simplified equation to predict glomerular filtration rate from serum creatinine. J Am Soc Nephrol 2000;11:155A (A0828).

[19] Gonzalez-Vilchez F, de Prada JA, Castrillo C, Canteli A, Llano MF, Martin-Duran R. Predictors of long-term renal function after conversion to proliferation signal inhibitors in longterm heart transplant recipients. J Heart Lung Transplant 2011; 30:552.

[20] Nankivell BJ, Borrows RJ, Fung CL, O’Connell PJ, Chapman JR, Allen RD. Calcineurin inhibitor nephrotoxicity: longitudinal assessment by protocol histology. Transplantation 2004;78:557.

[21] Navarro-Manchon J, Martinez-Dolz L, Almenar L, et al. Prognostic value of glomerular filtration rate 1 year after heart transplantation. Rev Esp Cardiol 2010;63:564.

[22] Crespo-Leiro MG, Delgado JF, Paniagua MJ, et al. Prevalence and severity of renal dysfunction among 1062 heart transplant patients according to criteria based on serum creatinine and estimated glomerular filtration rate: results from the CAPRI study. Clin Transplant 2010;24:E88.

[23] Gullestad L, Mortensen SA, Eiskjaer H, et al. Two-year outcomes in thoracic transplant recipients after conversion to everolimus with reduced calcineurin inhibitor within a multicenter, open-label, randomized trial. Transplantation 2010;90:1581. 
[24] Angermann CE, Stork S, Costard-Jackle A, et al. Reduction of cyclosporine after introduction of mycophenolate mofetil improves chronic renal dysfunction in heart transplant recipients-the IMPROVED multi-centre study. Eur Heart J 2004;25:1626.

[25] Manito N, Rabago G, Palomo J, et al. Improvement in chronic renal failure after mycophenolate mofetil introduction and cyclosporine dose reduction: four-year results from a cohort of heart transplant recipients. Transplant Proc 2011;43:2699.

[26] Groetzner J, Kaczmarek I, Schulz U, et al. Mycophenolate and sirolimus as calcineurin inhibitor-free immunosuppression improves renal function better than calcineurin inhibitorreduction in late cardiac transplant recipients with chronic renal failure. Transplantation 2009;87:726.

[27] Aliabadi AZ, Pohanka E, Seebacher G, et al. Development of proteinuria after switch to sirolimus-based immunosuppression in long-term cardiac transplant patients. Am J Transplant 2008; 8:854.

[28] Noris M, Mister M, Pezzotta A, et al. ACE inhibition limits chronic injury of kidney transplant even with treatment started when lesions are established. Kidney Int 2003;64:2253. 